\title{
JOS Special Issue on Responsive and Adaptive Survey Design: Looking Back to See Forward - Editorial
}

\author{
In Memory of Professor Stephen E. Fienberg, 1942-2016
}

\author{
Asaph Young Chun ${ }^{1}$, Barry Schouten ${ }^{2}$, and James Wagner $^{3}$
}

\section{Introduction}

A rapidly changing survey environment today requires a nimble, flexible design that leverages multiple data sources, frequently collects data in multiple modes, produces high quality data, and optimizes cost allocation around the survey life cycle. In this environment, surveys are ever more challenging and complex to implement. It was in response to these challenges that responsive and adaptive designs were born. This special issue includes twelve articles that give a glimpse of how these concepts are being used in diverse settings.

Three phenomena are worth noting. First, evidence-driven policy makers as well as survey researchers have renewed their attention to administrative records, as a key additional data source to enhance survey data quality and minimize survey cost (Chun 2009; Nordbotten 2010; Chun and Scheuren 2011; Chun and Larsen forthcoming). Big data, sometimes called organic data, also create new opportunities when linked to survey data. Second, the computerization of survey data collection has made accessible real-time analysis of paradata, or process data. Paradata have proven useful for managing existing surveys and designing future ones (Couper 1998; Chun and Kwanisai 2010; Couper and Kreuter 2012). Third, methods from fields as diverse as machine learning, operations research, and Bayesian statistics have been found to be useful for the advancement of response propensity modeling and in the design of creative surveys (Early, Mankoff and Fienberg in this special issue; Calinescu et al. 2013; Little 2006). Responsive and adaptive survey designs are evolving in this dynamic environment.

The JOS is publishing this special issue in memory of Professor Stephen Fienberg, University Professor of Statistics and Social Science at Carnegie Mellon University, who

${ }^{1}$ Asaph Young Chun is Research Chief for Decennial Directorate at the U.S. Census Bureau. Washington, D.C., 20233, USA. Email: Asaph.Young.Chun@ census.gov.

${ }^{2}$ Barry Schouten is Senior Methodologist at Statistics Netherlands and Professor at Utrecht University, The Netherlands. Email: jg.schouten@cbs.nl.

${ }_{3}^{3}$ James Wagner is Research Associate Professor at the University of Michigan, Ann Arbor, MI, 48109, USA. Email: jameswag@umich.edu.

Acknowledgments: We would like to extend our heartfelt thanks to the contributors to this Special Issue for their dedicated work and to all peer reviewers for their methodical review. We are also so grateful to Ingegerd Jansson, Susanna Emanuelsson and the JOS editorial staff who provided critical support.

Disclaimer: The views expressed are the authors' and not necessarily those of the institutions with which the authors are affiliated. 
passed away in December, 2016. An internationally acclaimed statistician, Fienberg was an interdisciplinary scholar of statistics, machine learning and computer science, an author and critical reviewer of numerous JOS articles, and a transformative contributor to JOS for several decades (e.g., Fienberg 1994; Fienberg and Makov 1998). In light of the increasing use of administrative data for statistical purposes and the attendant risk of disclosing confidential data, he spearheaded the 1998 JOS Special Issue on Disclosure Limitation Methods for Protecting the Confidentiality of Statistical Data (Fienberg and Willenborg 1998). A plenary speaker in the JOS 30th Anniversary Conference in 2015, Fienberg inspired the community of official statistics to bear the challenge of "Confidentiality in an era of Big Data." JOS is highly honored to publish in this special issue an article of adaptive design that Fienberg coauthored with Carnegie Mellon colleagues.

\section{Reflections on Research on Responsive and Adaptive Survey Designs}

The term responsive and adaptive survey design has been used for a decade (Groves and Heeringa 2006; Wagner 2008). In practice, these types of designs have been around much longer, if only without the labels. This history is logical, as the basic rationale of survey design is that different population subgroups react differently to different design choices in terms of response and measurement. Subgroups also vary in relation to costs and the key survey statistics. The idea to vary design feature choices over subgroups seems natural. A more systematic framework and approach to implementing this idea have evolved over the last decade (Laflamme and Karaganis 2010; Peytchev et al. 2010; Schouten et al. 2013). This evolution is likely due to three main reasons: an increasingly strong pressure on response rates (and thus costs), the emergence of data collection process data or paradata, and the evolution of new survey modes especially via the Internet. These changes make a much stronger business case for adaptation. There are potentially more levers and more clues, and there is more urgency.

Adaptive and responsive survey design are not well defined terms as they have emerged in different survey settings. There is a variety of alternative terms. Some may refer to the designs as targeted or tailored designs. Responsive survey design (Groves and Heeringa 2006) originates from settings with relatively little auxiliary data, long data collection periods and the desire to meet detailed quality-cost constraints even under incidental variation. Adaptive survey design (Wagner 2008) comes from settings where the focus is on richer auxiliary data, relatively shorter data collection periods and on structural variation. Terminology itself is less important than its substance. What these survey designs share are four key elements: auxiliary data, design features/interventions, explicit quality and cost metrics, and quality-cost optimization.

Considering the four key elements, there has been ample research on paradata (Kreuter 2013), indicators for monitoring nonresponse (Wagner 2012; Nishimura et al. 2016), design interventions in interviewer surveys (Walejko and Wagner 2015) and choice of mode (Calinescu and Schouten 2015, 2016). However, the interaction between auxiliary data and design features has received less investigation. We know little about how to identify subgroups and match different protocols to each group in order to improve quality or reduce costs. Research into optimization remains thin (Schouten and Shlomo 2017). 
Over the last ten years, the interest in adaptive and responsive survey design has greatly increased and a good deal of research has been presented at conferences and in journals (Tourangeau et al. 2016). This special issue is another proof of the lively interest in the designs. Actual implementations remain somewhat limited. The likely causes for this are threefold. First, the literature is to a large extent produced by survey statisticians and not by survey managers. There always has been a gap between data collection management and survey methodologists, even when it comes to non-adaptive survey designs. Adaptive and responsive survey design has a significant impact on survey logistics and processes and moving from theory to practice requires mutual understanding and common objectives. Second, the survey designs demand more complex monitoring and case management systems as well as explicit quality-cost objectives and norms. Finally, the number of success stories is limited. We hope this special issue may help fill in some of these gaps.

\section{Overview of the Special Issue}

This special issue highlights the variety of research being conducted under the umbrella of "responsive and adaptive design." Several articles provide formalized rules for adaptation. Paiva and Reiter in the present issue explore the potential impact of nonignorable nonresponse on estimates during data collection and use this approach to guide follow-up strategies. The article by Lewis builds on Groves and Heeringa's notion of "phase capacity" and develops decision rules for when to stop a phase of data collection. Early, Mankoff and Fienberg examine methods for dynamically re-ordering questionnaires in order to either maximize respondent engagement or, alternatively, to improve predictions concerning the respondent as quickly as possible. Vandenplas, Loosveldt and Beullens propose a new model-based method for comparing fieldwork progress across surveys. The method helps identify outlier events in the data collection process and may provide a signal of potential problems to survey managers. Burger, Perryck and Schouten propose and evaluate four perspectives to assess robustness of adaptive survey designs to inaccuracy of design parameter estimates.

Other articles examine the impact of responsive and adaptive designs on the quality of estimates. The article by Lundquist and Särndal is largely theoretical and investigates the impact of misspecification of regression coefficients on the utility and efficacy of adaptive survey design. Such coefficients can only be estimated for respondents and, hence, may suffer from bias. Re-examining the data compiled by Groves and Peytcheva (2008), Brick and Tourangeau in the present issue argue that there is an important between-study component of variance in addition to the within-study variance highlighted in the original analysis. The authors propose a theory implying that raising response rates can help reduce the nonresponse bias on average across the estimates within a study.

Three articles consider panel surveys or longitudinal designs where response data and paradata from prior waves are available. The article by Shlomo and Plewis shows how response propensity models can be used to improve the quality of response data in longitudinal studies. The authors use the U.K panel data to examine strategies for panel maintenance in longitudinal studies and improving the representativeness of the respondents over time through interventions. Lynn and Kaminska demonstrate how 
alternative methods for allocating sample elements to data collection protocols can be useful for panel surveys. The authors provide explicit quality and cost metrics to determine pros and cons of alternative methods tested. Durrant, Maslovskaya and Smith aim to address the question of how useful paradata, including call record data and interviewer observations, from the current and previous waves of a longitudinal study are in predicting response outcomes in future waves.

Two articles examine responsive and adaptive designs for establishment surveys. Thompson and Kaputa, and McCarthy, Wagner, and Sanders show how key elements of responsive and adaptive survey design can be tested and implemented in an establishment survey context. An experimental study design by Thompson and Kaputa allows the authors to investigate adaptive design relating to contact strategies for following up small establishments. A simulation study by McCarthy, Wagner, and Sanders explores methods for identifying subgroups for targeted designs in a survey of agricultural establishments for which there is a rich set of auxiliary data and historical paradata. The authors simulated different patterns of nonresponse to identify subgroups that may reduce nonresponse bias when their response propensities are changed.

\section{Remaining Challenges for Responsive and Adaptive Survey Design}

Responsive and adaptive design has a number of remaining challenges to address. The first challenge is to build the toolkit of tested designs that have been found to be effective. Many survey design features have been extensively investigated. However, we need to know more about how to deploy these features. First, we need to learn how to deploy them sequentially in ways that maximize quality and/or reduce costs, depending upon the priorities of the survey. The sequential deployment of features involves examining whether there are interactions between design features. For example, does following mail with web improve response rates relative to following web with mail? There is mixed evidence on this question (Wagner et al. 2014; Tourangeau 2017).

Second, we need to learn more about deploying features differentially across subgroups in the population. Although this idea has been around a long time, more evidence on specific strategies is needed. A general approach might be to offer high-responding subgroups the web mode while offering low-responding subgroups more expensive, intervieweradministered modes. Learning more about how various subgroups respond to the many varied design features is an essential input. Of course, every survey may need to define their subgroups differently, but building overlapping evidence is an essential part of this process.

Survey management and technical systems will need to evolve as well. Survey managers will need during the field period access to the latest information about costs, response rates, and data quality. This implies the development of monitoring tools that are flexible enough to adapt to changes in the survey environment while robust enough to provide accurate information that can be updated on a frequent basis. Survey designers need to develop auxiliary data or paradata that are relevant for the survey at hand. In order to be meaningful predictors of nonresponse bias, for example, these data have to be predictive of the survey outcome variables at hand.

Finally, survey managers will need to work more closely with survey statisticians. Designing responsive and adaptive designs will require experimentation and optimization 
techniques that are in the domain of survey statisticians. Survey statisticians, for their part, may need to borrow tools from operations research, machine learning, and other fields (e.g., Early, Mankoff and Fienberg in this special issue). Adaptive designs may require more complex optimization algorithms that may advance via the use of data science. A related challenge is developing rules for when to switch from one phase to another, or when to stop collecting data altogether (e.g., articles by Paiva and Reiter and by Lewis in this special issue). Using incoming data to update pre-survey estimates of design parameters may be facilitated by Bayesian approaches. While it is possible to improve the balance of response by lowering response rates for high-responding groups, or to save money in this way, we need to continue to challenge ourselves to find methods for improving data quality for the same or even lower costs.

We hope the articles featured in this special issue become a catalyst of further innovation in adaptive and responsive survey design.

Guest Editors Asaph Young Chun

Barry Schouten

James Wagner

\section{References}

Calinescu, M., S. Bhulai, and B. Schouten. 2013. "Optimal Resource Allocation in Survey Designs." European Journal of Operations Research 226(1): 115-121. Doi: https://doi.org/10.1016/j.ejor.2012.10.046.

Calinescu, M. and B. Schouten. 2015. "Adaptive Survey Designs to Minimize Mode Effects. A Case Study on the Dutch Labour Force Survey." Survey Methodology 41(2): 403-425.

Calinescu, M. and B. Schouten. 2016. “Adaptive Survey Designs for Nonresponse and Measurement Error in Multi-Purpose Surveys." Survey Research Methods 10(1): $35-47$.

Chun, Y. 2009. Nonparticipation of the 12th graders in the National Assessment of Educational Progress: Understanding Determinants of Nonresponse and Assessing the Impact on NAEP Estimates of Nonresponse Bias According to Propensity Models. University of Maryland, College Park, USA. Available at: http://hdl.handle.net/1903/ 9916 (accessed June 20, 2017).

Chun, Y. and M. Kwanisai. 2010. “A Response Propensity Modeling Navigator for Paradata.” In Proceedings of the Survey Research Methods Section of the American Statistical Association, 356-369. Joint Statistical Meetings, Vancouver, Canada. Available at: http://ww2.amstat.org/sections/SRMS/Proceedings/y2010/Files/306125_55196.pdf (accessed June 20, 2017).

Chun, Y. and M. Larsen. (forthcoming). Administrative Records for Survey Methodology, Edited book, Wiley Series in Survey Methodology, John Wiley \& Sons.

Chun, Y. and F. Scheuren. 2011. "Pandata Systems to Enhance Survey and Census Systems: Employing Administrative Data to Better Inform Public Policy." Invited Paper Presented at the Joint Statistical Meetings, Miami Beach, FL, USA. 
Couper, M.P. 1998. "Measuring Survey Quality in a CASIC Environment." In Proceedings of the Section on Survey Research Methods of the American Statistical Association, Washington, DC.

Couper, M.P. and F. Kreuter. 2012. "Using Paradata to Explore Item Level Response Times in Surveys.” Journal of the Royal Statistical Society: Series A 176: 271-286. Doi: http://dx.doi.org/10.1111/j.1467-985X.2012.01041.x.

Fienberg, S.E. 1994. "Conflicts Between the Needs for Access to Statistical Information and Demands for Confidentiality." Journal of Official Statistics 10: 115-132.

Fienberg, S.E. and U.E. Makov. 1998. "Confidentiality, Uniqueness and Disclosure Limitation for Categorical Data.” Journal of Official Statistics 14: 385-397.

Fienberg, S.E. and L.C.R.J. Willenborg. 1998. "Introduction to the Special Issue: Disclosure Limitation Methods for Protecting the Confidentiality of Statistical Data." Journal of Official Statistics 14: 337-345.

Groves, R.M. and S.G. Heeringa. 2006. "Responsive Design for Household Surveys: Tools for Actively Controlling Survey Errors and Costs." Journal of the Royal Statistical Society: Series A 169: 439-457. Doi: http://dx.doi.org/10.1111/j.1467-985X. 2006.00423.x.

Groves, R.M. and E. Peytcheva. 2008. "The Impact of Nonresponse Rates on Nonresponse Bias - a Meta-Analysis." Public Opinion Quarterly 72(2): 167-189. Doi: https:// doi.org/10.1093/poq/nfn011.

Kreuter, F. 2013. Improving Surveys with Paradata. Analytic Use of Process Information. Edited book, Wiley Series in Survey Methodology, John Wiley \& Sons.

Laflamme, F. and M. Karaganis. 2010. "Implementation of Responsive Collection Design for CATI Surveys at Statistics Canada.” Paper presented at Q2010, 3-6 May, Helsinki, Finland.

Little, R.J.A. 2006. "Calibrated Bayes: A Bayes/Frequentist Roadmap." The American Statistician 60(3): 213-223. Doi: http://dx.doi.org/10.1198/000313006X117837.

Nishimura, R., J. Wagner, and M.R. Elliott. 2016. "Alternative Indicators for the Risk of Non-Response Bias: A Simulation Study." International Statistical Review 84(1): 43-62. Doi: http://dx.doi.org/10.1111/insr.12100.

Nordbotten, S. 2010. "The Use of Administrative Data in Official Statistics - Past, Present, and Future - with Special Reference to the Nordic countries." Official Statistics in Honour of Daniel Thorburn, 205-223. Norway.

Peytchev, A., S. Riley, J. Rosen, J. Murphy, and M. Lindblad. 2010. "Reduction of Nonresponse Bias in Surveys Through Case Prioritization." Survey Research Methods 4(1): 21-29.

Schouten, B., M. Calinescu, and A. Luiten. 2013. "Optimizing Quality of Response Through Adaptive Survey Designs.” Survey Methodology 39(1): 29-58.

Schouten, B. and N. Shlomo. 2017. "Selecting Adaptive Survey Design Strata with Partial R-Indicators.” International Statistical Review 85(1): 143-163. Doi: http://dx.doi.org/ 10.1111/insr.12159.

Tourangeau, R. 2017. "Mixing Modes: Tradeoffs among Coverage, Nonresponse, and Measurement Error." In Total Survey Error in Practice, edited by P. Biemer, E. de Leeuw, S. Eckman, B. Edwards, F. Kreuter, L.E. Lyberg, N.C. Tucker, and B. West, New York: Wiley. 
Tourangeau, R., M. Brick, S. Lohr, and J. Li. 2016. "Adaptive and Responsive Survey Designs: a Review and Assessment." Journal of the Royal Statistical Society: Series A 180: 203-223. Doi: http://dx.doi.org/10.1111/rssa.12186.

Wagner, J. 2008. “Adaptive Survey Design to Reduce Nonresponse Bias.” PhD thesis, University of Michigan, Ann Arbor, USA.

Wagner, J. 2012. "A Comparison of Alternative Indicators for the Risk of Nonresponse Bias.” Public Opinion Quarterly 76(3): 555-575. Doi: https://doi.org/10.1093/ $\mathrm{poq} / \mathrm{nfs} 032$.

Wagner, J., J. Arrieta, H. Guyer, and M.B. Ofstedal. 2014. "Does Sequence Matter in Multimode Surveys: Results from an Experiment." Field Methods 26(2): 141-155. Doi: http://dx.doi.org/10.1177/1525822X13491863.

Walejko, G. and J. Wagner. 2015. "Challenges to Innovation in Face-to-Face Surveys Posed by Interviewer Noncompliance.” Paper presented at the American Association for Public Opinion Research, May 14-17, Hollywood, FA, USA. 